\begin{tabular}{ccc}
\hline International Journal of Health Services \\
Research and Policy \\
www.dergipark.org.tr/ijhsrp \\
INTERNATIONAL \\
ENGINEERING \\
SCIENCE AND \\
EDUCATION GROUP
\end{tabular}

Research Article

\title{
HEALTH WORKER AND CAREGIVER INTERACTION DURING CHILD VACCINATION SESSIONS AT HEALTH FACILITIES IN SOMALI REGION OF ETHIOPIA: A QUALITATIVE STUDY
}

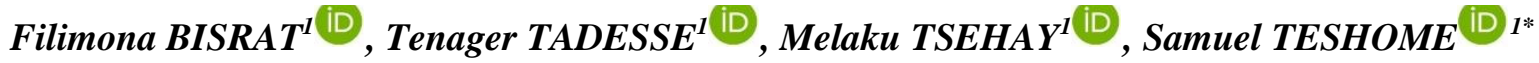 \\ ${ }^{1}$ CORE Group Polio Project, Akaki sub-city, Addis Ababa: P. O. Box 5674, Ethiopia \\ *corresponding author; samteshome@gmail.com
}

\begin{abstract}
Somali Region is one of the largest regions in Ethiopia which has performed persistently low with immunization coverage. Communication during vaccination sessions at health facilities is a key factors that influence caregiver's decision to bring back their children for vaccination. The objective of this study was to assess health worker and caregiver interaction during immunization sessions and identify communication gaps at health facilities in the Somali region. This was a cross-sectional qualitative study. The study was carried out using in-depth interviews with health workers responsible for vaccination, observation of vaccinator and caregiver interaction during immunization sessions, and exit interview of caregivers who brought their children for vaccination or whose child was vaccinated at the health facility. Health workers responsible for vaccination in twelve health facilities from the central and remote parts of the region were interviewed. A total of 63 vaccination sessions in the 12 health facilities were observed and caregivers were interviewed on exit. Most caregivers described the vaccinators as friendly and supportive. Some mothers who did not bring the immunization card with them were returned without the child getting the service. The return date for vaccination was written on child immunization card in most cases. Most caregivers do not identify vaccines by name or purpose, however, they all want their children to get vaccines as recommended by health workers. Health workers do not communicate all information required for continued use of immunization services at health facilities during vaccination sessions. In the Somali region, caregiver communication will need to be improved through training of health workers on immunization basics and effective communication skills. Keywords: vaccination, health worker, communication, Ethiopia
\end{abstract}

Received: December 31, 2019

Accepted: June 02, 2020

\section{Introduction}

Globally two to three million children die of vaccine-preventable diseases annually [1]. Immunization remains a reliable high impact strategy to decrease deaths from vaccine-preventable diseases and a key strategy to attain sustainable development goal 3 that aims to decrease mortality in under-fives to 25/1000 life births by 2030 [2-4]. Immunization has enabled disease eradication and elimination and substantial gain in improved child survival [5]

The national immunization program in Ethiopia includes BCG and Polio vaccine given at birth, 
the DPT-HepB- HiB (Pentavalent vaccine) OPV, PCV vaccines given at age 6, 10, and 14 weeks. Measles containing vaccine given at 9 months and repeated after 6 months, Rota vaccine is given at age 6 and 10 weeks while IPV is given at age 14 weeks. The HPV vaccine was introduced in 2019 and is given to girls at 14 years of age [6].

The Ethiopian Demographic Health Survey (EDHS) has shown consistent improvement in national EPI coverage over the past years from $18 \%$ in 2000 to $39 \%$ in 2016. The current highest vaccine coverage is in Addis Ababa (89\%) and the lowest in the Somali region (19\%) [7].

The Somali region located in the east southeastern part of Ethiopia is one of the regions that has persistently performed low with routine immunization coverage. In 2018 the region administrative data for Penta 3 coverage was $71 \%$ and measles coverage $67 \%$, way below the national target of $90 \%$. The dropout rate from Penta 1 to Penta 3 was $15.6 \%$, which is above the highest acceptable level of $10 \%$. [8].

Communication is one of many interacting factors that influence parents' and other caregivers' decisions to take their children for vaccination. Communication alone cannot address all aspects of vaccine hesitancy or refusal. However, communication interventions are an important component of vaccination and inadequate communication can hurt vaccination uptake, vaccine completion, and parental trust in a vaccination program (9). In most settings, communication about childhood vaccination is common, but there is uncertainty around how people perceive and understand communication from health workers at health facilities during vaccination sessions and whether and how this influences their decision to vaccinate. Understanding and improving health worker/caregiver interactions can lead to health workers' gaining more satisfaction from their vaccination work and caregivers feeling more satisfied and better informed, and more children being fully vaccinated and protected (9-10) Health worker and caregiver communication during vaccination sessions is one of the important factors, along with health worker's knowledge, attitude and practice that determine successful immunization service uptake. A Study evaluating health worker and caregiver interaction at health facilities during vaccination session and gaps in communication to our knowledge is nonexistent

The objective of this study was, therefore to assess health worker and caregiver interaction during vaccination sessions at health facilities in the Somali region and to identify gaps in communication that contribute to low completion of immunization.

\section{Material and Methods}

\subsection{Study site}

The study was conducted in the Somali Region of Ethiopia. The region has a population of 6 million with a majority (85\%) moving or pastoralist population. The region has 1 central hospital, 3 general hospitals, 9 primary hospitals 216 health centers, and 1311 health posts. The Somali Region is subdivided into eleven administrative zones, which are subdivided into 93 districts (Woredas) and six town administrations, and further subdivided into sub-districts (Kebele), which are the lowest administrative unit [11].

\section{Study design:}

The study used a qualitative cross-sectional method. In-depth interview of health workers at health facilities and exit interview of caregivers after vaccination session was conducted using a semistructured questionnaire. All vaccination sessions on the day of the visit were observed. 


\subsection{Respondent sample}

Three districts in the region, geographically representing central and remote areas were selected purposively. In the district, health facilities were listed and a health center and a health post were selected randomly. At each health facility, an in-depth interview of health workers responsible for vaccination, observation of vaccinator and caregiver interaction during immunization session, and exit interview of caregiver after the vaccination session was done.

\subsection{Assessment of health worker and caregiver interaction}

In-depth interviews, using in-depth interview guides, on health workers' general levels of motivation regarding their immunization responsibilities, vaccine and vaccination knowledge, service delivery, communication with caregivers, and collaboration with community volunteers to pass on immunization messages was conducted. Data collectors were selected based on their knowledge of the local language and previous experience in qualitative research. All data collectors were trained on the study tools. A team consisting of an interviewer and a note-taker conducted an in-depth interview with health workers. Interview proceedings were recorded.

Health worker and caregiver interaction during immunization sessions in each health facility were documented through observation. After observation, an exit interview of caregivers whose child received vaccination at the health facility was conducted. Interview guides for an exit interview and a checklist for observation of vaccination sessions were used. During observation and exit interview, caregivers waiting time at a health facility, their experience of the vaccination session, caregiver's knowledge of the return date and possible side effects, use of immunization card, and caregiver's experience with the health worker during the vaccination session were assessed.

\subsection{Data management}

Findings from in-depth interview audio recordings were transcribed into Microsoft word 2010 by the data collectors who were fluent in the local language and English. After transcription, the transcribed document was reviewed by investigators and compared to note taken in the field for accuracy. The investigators coded the transcripts utilizing focused coding based on the objectives of the study. The codes were then entered into qualitative analysis software ATLAS.ti 8 by topic to explore both anticipated and emergent themes using a thematic analysis technique.

\section{Ethical considerations}

The Somali region health bureau ethical committee reviewed and approved the study. Ethical clearance for the study was obtained from the ethics committee of the Somali region health bureau. (Certificate permit number, SRHB/18/2000-2005 on 20/6/19). Informed consent was obtained from each participant for the in-depth interview, observation, and exit interview. The observation was conducted after obtaining informed consent both from the health worker and caregiver.

\section{Results and Discussion}

The qualitative study was conducted in the Somali region between 20-26 July 2019. The study enrolled and analyzed in-depth interviews from health workers in twelve selected health facilities in six districts. Most of the vaccinators at health centers were nurses while health extension workers provided vaccination service at health posts (Table 1). 
Table 1. In-Depth Interviews: Details of health workers interviewed

\begin{tabular}{|c|c|c|c|c|c|c|}
\hline Zone & Facility & Profession & Sex & $\begin{array}{c}\text { Year } \\
\text { of } \\
\text { service }\end{array}$ & Main task & Other tasks \\
\hline \multirow[t]{4}{*}{ Siti } & Error HC & Nurse & $\mathrm{F}$ & 6 & Vaccinator & MCH/Family planning \\
\hline & $\begin{array}{c}\text { Kanteras Health } \\
\text { Post }\end{array}$ & Nurse & $\mathrm{F}$ & 5 & Vaccinator & $\begin{array}{c}\text { The Health extension } \\
\text { package }\end{array}$ \\
\hline & $\begin{array}{l}\text { Hurso Health } \\
\text { center }\end{array}$ & Nurse & M & 3 & Epi focal person & $\begin{array}{c}\text { Cold Chain and Vaccine } \\
\text { management }\end{array}$ \\
\hline & $\begin{array}{c}\text { Halisho Health } \\
\text { Post }\end{array}$ & Nurse & $\mathrm{F}$ & 5 & $\begin{array}{l}\text { Health extension } \\
\text { worker }\end{array}$ & Health extension package \\
\hline \multirow[t]{4}{*}{ Liban } & $\begin{array}{l}\text { Aynle health } \\
\text { Center }\end{array}$ & Nurse & $\mathrm{F}$ & 5 & vaccinator & MCH/Family Planning \\
\hline & $\begin{array}{l}\text { Hysuftu Health } \\
\text { center }\end{array}$ & Nurse & M & 5 & Epi Focal person & Health extension package \\
\hline & $\begin{array}{l}\text { Mesjidi Health } \\
\text { post }\end{array}$ & Nurse & $\mathrm{F}$ & 5 & Epi Focal person & HEW supervisor \\
\hline & $\begin{array}{l}\text { Dheka Suftu } \\
\text { Health Post }\end{array}$ & HEW & M & 1 & Vaccinator & Health extension package \\
\hline \multirow[t]{4}{*}{ Shebele } & $\begin{array}{l}\text { Adade health } \\
\text { center }\end{array}$ & Nurse & $\mathrm{F}$ & 4 & Epi Focal person & MCH/Family planning \\
\hline & $\begin{array}{l}\text { Adade Health } \\
\text { Post }\end{array}$ & HEW & M & 9 & Vaccinator & Health extension package \\
\hline & $\begin{array}{l}\text { Kelafo health } \\
\text { center }\end{array}$ & Nurse & $\mathrm{F}$ & 9 & Epi Focal Person & MCH/Family Planning \\
\hline & $\begin{array}{c}\text { Hillobad Health } \\
\text { Post }\end{array}$ & HEW & $\mathrm{F}$ & 1 & Vaccinator & Health Extension package \\
\hline
\end{tabular}

A total of 63 vaccination sessions in twelve health facilities were observed, and caretakers with a child vaccinated at health facility were interviewed on exit. All of the caretakers who brought a child to health facility were mothers and the majority were in the age group 25-34 years $(52 \%, 33 / 63)$ and most can not read and write (54\% 34/63) (Table 2).

Table 2. Profile of Caregivers Who Brought Child to Health Facility for Vaccination

\begin{tabular}{|c|c|c|c|c|c|c|c|c|c|}
\hline \multirow[t]{2}{*}{ Caregiver } & \multicolumn{3}{|c|}{ Age } & \multicolumn{2}{|c|}{ Relation to child } & \multirow{2}{*}{$\begin{array}{c}\text { Language } \\
\text { Somali }\end{array}$} & \multirow[b]{2}{*}{ Other } & \multicolumn{2}{|c|}{ Read and Write } \\
\hline & $15-24$ & $25-35$ & $36-49$ & Mother & Other & & & Yes & No \\
\hline Number & 29 & 33 & 1 & 63 & 0 & 62 & 1 & 29 & 34 \\
\hline Percentage (\%) & 46 & 52 & 2 & 100 & 0 & 98 & 2 & 46 & 54 \\
\hline
\end{tabular}

\subsection{Findings from the in-depth interview}

The main themes that emerged surrounding health worker's motivation and practice were health workers job satisfaction, knowledge on vaccine and vaccine contraindication, vaccination card usage, challenges in service delivery, and collaboration with community volunteers.

Despite working in difficult circumstances, with insufficient support and compensation and supply of vaccine or other essential commodities there was a high level of motivation from health workers providing immunization. 
All vaccinators at the health facilities were happy on the work they do to get children vaccinated as the respondents below said:

"I enjoy vaccinating children and there are no aspects of the immunization work I do that I do not like" (Health extension worker, at Dekasuftu health post).

The decision by vaccinator when caregivers forget to bring the immunization card with them is mixed. Some tell the caregivers to go back and bring the card, especially if the residence is nearby, and do not give the vaccine to the child. Others advise them to bring for the next visit and give the vaccine to the child after checking the child's records on the immunization registration book at the facility.

All vaccinators responded that for those caregivers who bring immunization cards with them they give vaccines to the child even if the due date the child was supposed to get the vaccine has passed. "When caregiver brings child late for vaccination, I do not have a bad feeling towards the caretaker, I ask the caretaker the reason for not coming on the date of appointment, then check the vaccination card and advise them that vaccine should be taken on the date specified and not to be late again and give the vaccine required to the child" (EPI focal person and vaccinator: Hurso health center).

"When mothers forget to bring the immunization card, I send them back to bring the card, it is very difficult for me to give service to a child without an immunization card." (Epi focal person and vaccinator: Adade Health Center, Shebele Zone).

Most health workers mentioned that children aged one year and below are target groups for routine immunization and provide the certificate of completion to children who completed the primary series at 9 months of age. At the time they provide the certificate of completion however, they tell caregivers to bring their child at 15 months of age for the MCV2 vaccine.

A sick child is commonly mentioned by the health worker as a contraindication for vaccine. However, there was a lack of clarity on the degree of sickness that should be a contraindication to give a vaccine. Most health workers know that a child might develop fever and pain at the injection site however, this information is not regularly passed on to caregivers.

All mentioned that their facilities conduct vaccination sessions only twice weekly at health centers and weekly at health posts, and in all facilities, BCG and Measles vaccines are given monthly. Health workers mentioned that the vaccination session schedule is based on caregivers' convenience, shortage of BCG and measles vaccine, and facility decision to offer these vaccines on fewer days to avoid vaccine wastage. All health centers conduct outreach sessions; however, outreach sessions are not conducted regularly due to a shortage of transport services. Some felt that their facilities do not have adequate staff and most of the health centers and all health posts involve community volunteers in their activities and consider their contribution as the most important single factor for a successful vaccination program.

\subsection{Findings from Observation of vaccination sessions}

Most caregivers (87\% 120/138) brought immunization card with them when they visited health centers than those visiting health posts $(57 \%, 79 / 138)$. Vaccinators in the majority of the health facilities mentioned the return date and its importance to caregivers. However, there was inadequate documentation of the return date and vaccine given on immunization cards. Most children visiting health centers received all the vaccine they required than those at health posts (Figure 1). 


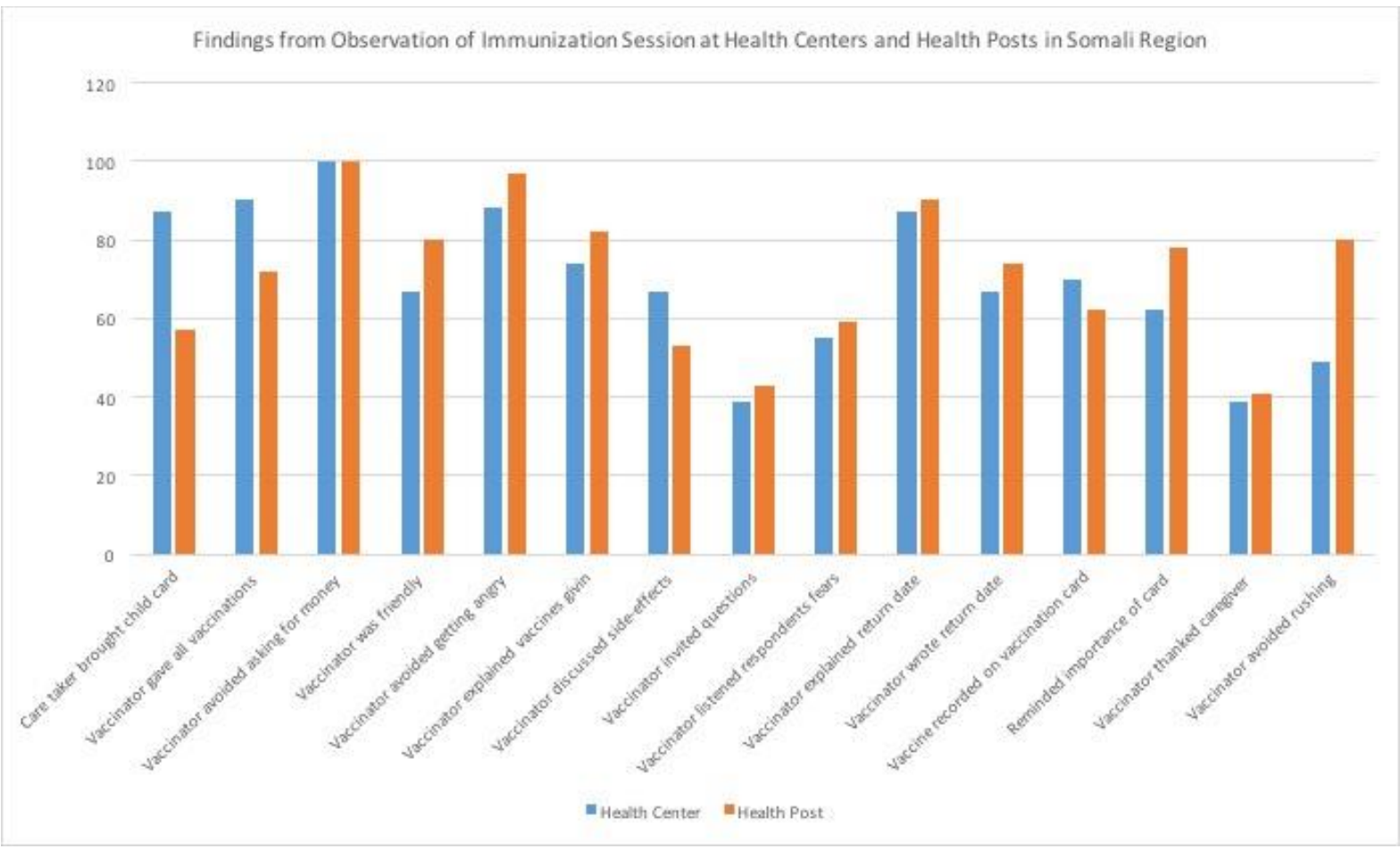

Figure 1.Finding from observation of immunization session at health centers and health posts in the Somali region

\subsection{Findings from Exit Interview}

The average time that a caregiver stays at the health facility for the child to be vaccinated is higher at health posts (average 20.8 minutes, range 15-35 minutes) than at health centers, (average 11.6 minutes, range 10-15minutes), (Figure 2). All caregivers praised health workers' treatment during the service. Caregivers identify the vaccine given to the child by the site of injection and route of administration and not by name and purpose. They consider vaccines to be important to prevent illness. Most caregivers brought the immunization card with them however, only $67 \%$ of the cards at health centers and $74 \%$ at the health posts had a return date written. Most caregivers correctly mentioned the return date as told by the health worker, some did not. Most caretakers responded that fever and pain at the injection site are possible expected side effects and mentioned this is from their previous experience and not because it was told by the health worker. 


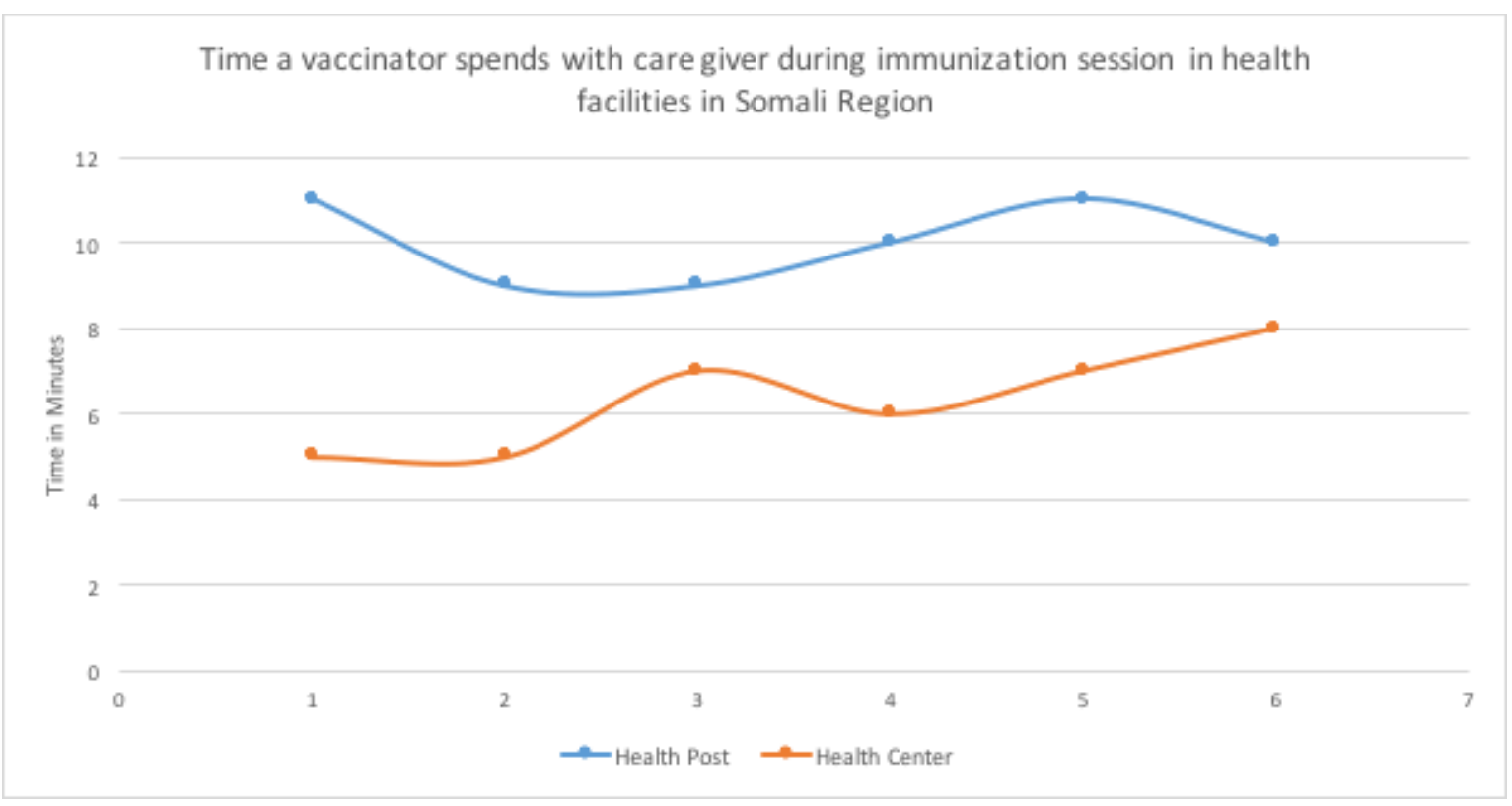

Figure 2. Time vaccinator spends with the caregiver during immunization session in Somali region

\section{Discussion}

In this cross-sectional study, we found that the quality of interaction between the health worker and caregiver during immunization sessions at health centers and health posts in the Somali region is affected by the following factors.

There is a high level of illiteracy among caregivers who brought children for vaccination. Caregivers who can not read will not comprehend what is written, and the purpose and importance of the child vaccination card. Consequently, some caregivers do not bring the child vaccination card with them, and some vaccinators do not give service and return back the caregiver to bring the card. This could be avoided if the contents and purpose of the immunization card are clearly and regularly communicated with caregivers and advised to bring with them when they visit the health facility.

Health workers in the Somali region are characterized as friendly by caregivers and avoided getting angry when there was a reason to be. This is a very positive and encouraging finding and departure from finding of other studies [12-16] that suggested health worker's attitude was a major hindrance to caregiver uptake of immunization services. A bad experience may affect caregiver willingness to vaccinate subsequent children and caregivers may discuss their negative experience with others in the community. This is also reflected in Demographic and Health Survey data that shows higher birth order children tend to receive fewer vaccinations [8].

Return date is not written on vaccination card in some cases and vaccinators verbally communicate the return date without confirmation that it is understood. In the majority of times, the caregiver forgets because the information was not captured or the caregiver expects the information on the return date to be told again sometime in the future. This finding is in agreement with the previous study done in Wonago, Ethiopia [17].

Caregivers did not know the specific vaccine that was administered to their child, this is because they could not read the name of the vaccine given from the vaccination card, however, this does not 
seem to be a factor in vaccine uptake as most caregivers believe that vaccination is good for their child. Similar findings were seen from studies in the Gambia [18] and Rwanda [19].

The time that the vaccinator interacts with the caregiver is brief, especially at health centers. This will have an impact on the scope and depth of information shared with the caregiver Vaccinators do not often communicate the side effects of the vaccine the child received although most caregivers mentioned that they know fever and pain at the injection site are expected after vaccination. A caregiver who does not have previous experience could be alarmed if side effects that the health worker failed to mention occur and could be a reason to keep a child away from vaccination [20].

The MCV2 is now added to the routine immunization schedule to be given 6 months after MCV1 which is given at age 9 months. Therefore, the certificate of completion of the basic vaccination should be given after children receive the MCV2 and not at 9 months as seen in some health facilities.

Contraindication for giving a vaccine in some facilities includes any sick child, this could be because of fear that health workers might be blamed if an illness gets worse after vaccination [21].

All vaccines are not available all day at health facilities. Notably BCG and MCV vaccine. The BCG and MCV vaccine are often provided monthly which requires the caregivers to come back on a separate schedule to get the service. In situations where the return on the specific date is not possible this will result in the child missing the vaccines.

Community volunteers contribute hugely to the success of immunization programs. The health facilities collaborate with the community volunteers to pass on immunization messages to the community, trace defaulters and pregnant mothers and notify newborns for vaccination. Involving community volunteers in vaccination programs has been documented as an important aspect of the success of immunization programs.

\section{Conclusion}

The study triangulated findings from in-depth interviews, observations, and exit interviews. The findings were found to be consistent irrespective of the method used. During the immunization session, health workers do not communicate all messages required for continued use of immunization services by the caretaker at health facilities. Caregiver interaction in the Somali region will need to be improved through training of health workers on the basics of immunization and Interpersonal communication (IPC). To standardize expectations and performance at health facilities there is a need to develop standard communication competency guides.

\section{Acknowledgments}

We are thankful to the health workers and caregivers who took their time to participate in the in-depth and exit interviews. We also thank the data collectors, the Somali Region Health Bureau, all study area health facility heads, and the CGPP admin and Finance team, who made this study possible. This study was supported by USAID through a grant to the CORE Group Polio Project.

\section{Ethical considerations}

The Somali region health bureau ethical committee reviewed and approved the study. Ethical clearance for the study was obtained from the ethics committee of the Somali region health bureau. (Certificate permit number, SRHB/18/2000-2005 on 20/6/19). Informed consent was obtained from each participant for the in-depth interview, observation, and exit interview. The observation was conducted after obtaining informed consent both from the health worker and caregiver. 
The compliance to the Research and Publication Ethics: This study was carried out in accordance with the rules of research and publication ethics.

\section{References}

[1] Casey, R et al. "Global routine vaccination coverage", Wkly. Epidemiol. Rec, 91, 537-548, 2016.

[2] Wolfson LJ et al. "Estimating the costs of achieving the WHO-UNICEF Global Immunization Vision and Strategy, 2006-2015”, Bulletin of the World Health Organization, 86(1), 27-39, 2008. https://doi.org/10.2471/BLT.07.045096 PMID: 18235887

[3] Maurice JM., Davey S. "State of the World's Vaccines and Immunization", Report. World Health Organization; 2009.

[4] Rutherford ME, et al. "Preventive measures in infancy to reduce under-five mortality: a casecontrol study in The Gambia", Tropical Medicine \& International Health, 14(2), 149-55, 2009.

[5] World Health Organization. "WHO vaccine-preventable diseases: monitoring system". Global summary, 2010.

[6] Federal Ministry of Health. "Ethiopia National Expanded Program on Immunization, Comprehensive Multi-Year Plan 2016-2020, Addis Ababa”, FMOH April 2015.

[7] Ethiopian Public Health Institute and ICF. "Ethiopia Mini Demographic and Health Survey", EDHS 2019.

[8] CSA Ethiopia and ICF. "Ethiopia Demographic and Health Survey; Key Indicators Report, Addis Ababa, Ethiopia and Rockville, Maryland, USA”. DHS: 27-28, 2016.

[9] Sawhney M, Favin, M. "Epidemiology of the unimmunized child. Findings from the grey literature, Arlington, VA”, IMMUNIZATION Basics Project, 2009.

[10] Favin M, et al. "Why children are not vaccinated: a review of the grey literature", International Health, 4, 229-38, 2012.

[11] Federal Ministry of Health: "Health Sector Transformation plan: Woreda Based Health Sector Annual Core Plan”, FMOH 2019-2020.

[12] Ministry of Health, UNICEF/Ethiopia. "National Immunization KABP Survey Report, Addis Ababa, Ethiopia”, 2001 [unpublished]

[13] Razum O. "Mothers voice their opinion on immunization services". World Health Forum, 14, 282-6, 1993.

[14] Perry, HB, et al. "The quality of urban EPI services in Bangladesh: findings from the Urban Initiatives' Needs Assessment Study in Zone 3 of Dhaka City, Dhaka, Bangladesh", ICDDR, B, Working Paper No. 24, 1996.

[15] UNICEF: "Social-Cultural Context of Immunization in Benin", Evaluation Newsletter [UNICEF] 12, 5, 1991. 
[16] Millimouno D, et al. "The social dynamics of infant evaluation in Africa, perspectives from the Republic of Guinea, Brighton, UK", Institute for Development Studies, University of Sussex, Working Paper, 262, 2006.

[17] Tadesse H, et al. "Explorative assessment of factors affecting child immunization in Wonago district, Gedeo zone, South Ethiopia”, Archives of Medical Science, 5(2), 233-40, 2009.

[18] Leach M., Fairhead J. "Understandings of immunization: some West African perspectives”, Bull World Health Organ, 86, 418, 2008.

[19] Habimana P., Bararwandika A. "Knowledge, attitudes and behavior of parents concerning immunization”, Imboneza- muryango/Famille, Sante, Development, 20, 8-13, 1991.

[20] M. Favin et al. "Why children are not vaccinated: a review of the gray literature", International Health, 4, 229-238, 2012.

[21] World Health Organization. "Epidemiology of the unvaccinated child: Findings from the gray Literature", WHO, October 2009. 